\title{
Determination of Temperature-Dependent Stress State in Thin AlGaN Layer of AlGaN/GaN HEMT Heterostructures by Near-Resonant Raman Scattering
}

\author{
Yanli Liu, ${ }^{1}$ Xifeng Yang, ${ }^{1,2}$ Dunjun Chen, ${ }^{1}$ Hai Lu, ${ }^{1}$ Rong Zhang, and Youdou Zheng ${ }^{1}$ \\ ${ }^{1}$ Key Laboratory of Advanced Photonic and Electronic Materials, School of Electronic Science and Engineering, Nanjing University, \\ Nanjing 210093, China \\ ${ }^{2}$ Jiangsu Laboratory of Advanced Functional Materials and College of Physics and Engineering, Changshu Institute of Technology, \\ Changshu 215500, China
}

Correspondence should be addressed to Dunjun Chen; djchen@nju.edu.cn

Received 28 November 2014; Accepted 17 December 2014

Academic Editor: Wen Lei

Copyright (C) 2015 Yanli Liu et al. This is an open access article distributed under the Creative Commons Attribution License, which permits unrestricted use, distribution, and reproduction in any medium, provided the original work is properly cited.

\begin{abstract}
The temperature-dependent stress state in the $\mathrm{AlGaN}$ barrier layer of $\mathrm{AlGaN} / \mathrm{GaN}$ heterostructure grown on sapphire substrate was investigated by ultraviolet (UV) near-resonant Raman scattering. Strong scattering peak resulting from the $A_{1}$ (LO) phonon mode of $\mathrm{AlGaN}$ is observed under near-resonance condition, which allows for the accurate measurement of Raman shifts with temperature. The temperature-dependent stress in the AlGaN layer determined by the resonance Raman spectra is consistent with the theoretical calculation result, taking lattice mismatch and thermal mismatch into account together. This good agreement indicates that the UV near-resonant Raman scattering can be a direct and effective method to characterize the stress state in thin AlGaN barrier layer of AlGaN/GaN HEMT heterostructures.
\end{abstract}

\section{Introduction}

Recently, AlGaN/GaN heterostructures have attracted considerable attention due to their potential use in high-power, high-temperature, and high-frequency electronic devices [1-5]. The high-temperature application is one important advantage of the AlGaN/GaN-based devices over GaAsbased and Si devices [6-8]. It is well known that the strain and stress in the AlGaN barrier layer due to lattice mismatch (LMM) and thermal mismatch between $\mathrm{AlGaN}$ and the underlying layers have important effect on the formation and transport properties of two-dimensional electron gas (2DEG) in AlGaN/GaN heterostructures [9-11]. Therefore, the investigation on the temperature dependence of stress or strain in AlGaN barrier layer is necessary for understanding the temperature-dependent electrical properties of AlGaN/GaN heterostructure and improving the reliability of the $\mathrm{AlGaN} / \mathrm{GaN}$ based devices.
In previous reports, the strain or stress in the $\mathrm{AlGaN}$ layer of $\mathrm{AlGaN} / \mathrm{GaN}$ heterostructures was characterized typically by using X-ray diffraction $[12,13]$. However, the reflection peaks of some asymmetric planes in AlGaN barrier layer are always invisible due to the thin thickness and poor interference of the plane [12]. So, the in-plane lattice constant and the biaxial strain of AlGaN layer cannot be measured directly using this method. Raman spectroscopy is an effective method for the residual stress measurement of crystal films. However, in the prior studies on Raman measurements of $\mathrm{AlGaN} / \mathrm{GaN}$ heterostructures, the visible $(532 \mathrm{~nm}, 488 \mathrm{~nm})$ Raman spectroscopy is mainly used to detect the stress and 2DEG channel temperature by measuring the phonon frequency of $\mathrm{GaN}$ averaged over the whole buffer layer $[14,15]$, which cannot reflect directly the stress state in the AlGaN barrier layer.

In this work, we investigated the temperature-dependent stress state in the thin AlGaN barrier layer of $\mathrm{AlGaN} / \mathrm{GaN}$ 


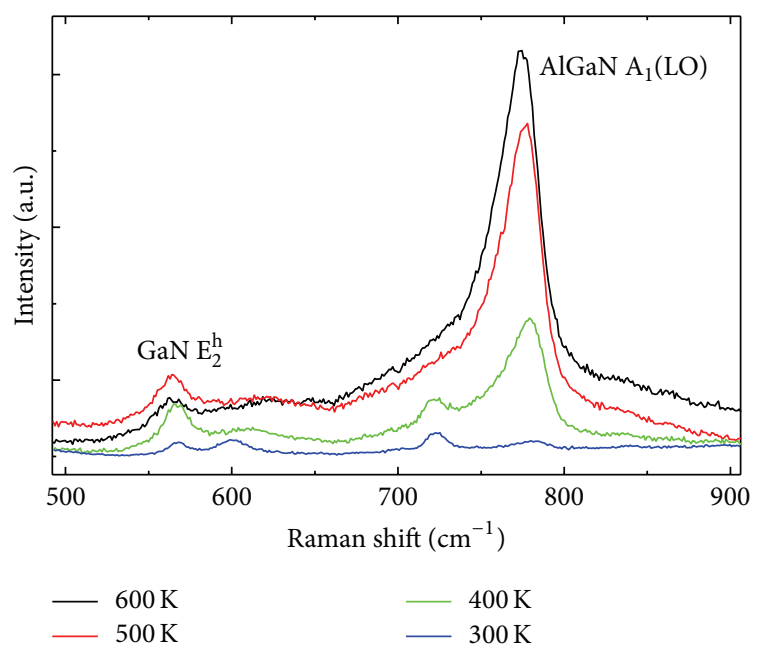

(a)

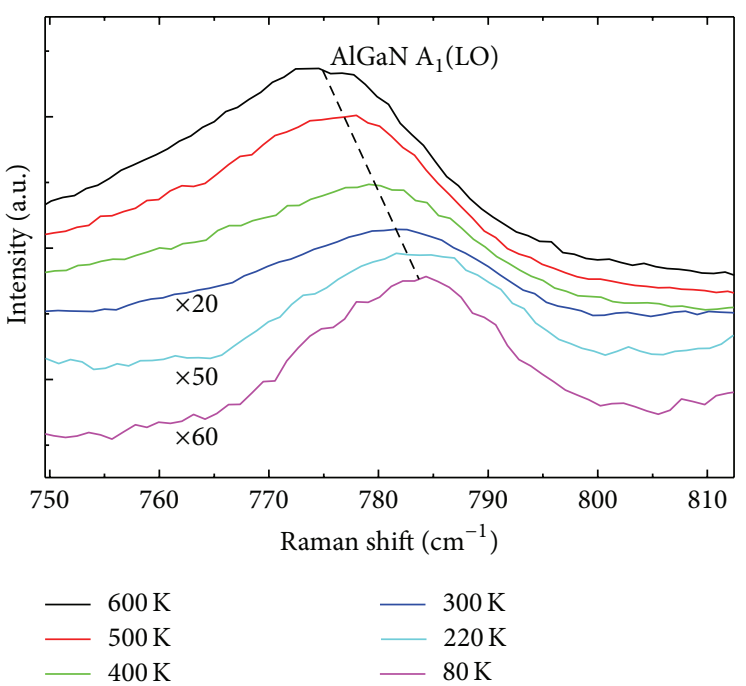

(b)

FIGURE 1: (a) UV Raman spectra of AlGaN/GaN heterostructure with varying temperature and (b) the local amplified graph of the $\mathrm{A}_{1}(\mathrm{LO})$ mode of AlGaN.

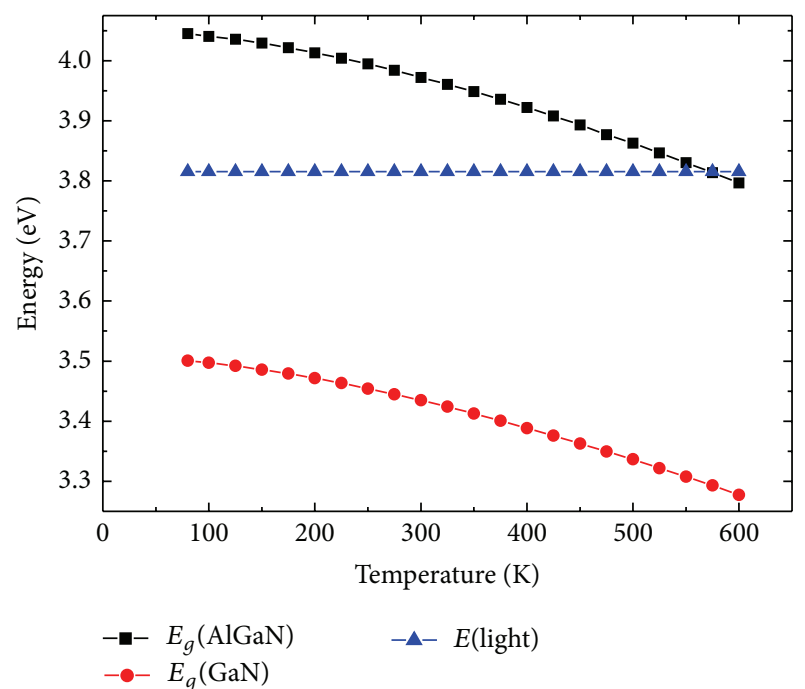

FIGURE 2: Temperature-dependent band gaps of the AlGaN/GaN heterostructure.

heterostructure by means of UV near-resonant Raman scattering. The Raman measured results are in good agreement with those from theoretical calculation, taking LMM and thermal mismatch into account together.

\section{Experiment}

The AlGaN/GaN heterostructure used in this study was grown on sapphire substrate by metal-organic chemical vapor deposition. The sample consists of a $2 \mu \mathrm{m}$ thick unintentionally doped GaN layer, a $1 \mathrm{~nm}$ thick AlN spacer layer, and a $25 \mathrm{~nm}$ thick undoped $\mathrm{Al}_{0.27} \mathrm{Ga}_{0.73} \mathrm{~N}$ barrier layer.
The Raman scattering spectra were recorded by using an HR800 Jobin-Yvon spectrometer equipped with a liquidnitrogen-cooled charge-coupled device in a backscattering geometry. A $325 \mathrm{~nm}$ He-Cd laser was used as an excitation source. A temperature stage with a quartz window was used to heat the sample from 80 to $600 \mathrm{~K}$ in flowing nitrogen.

\section{Results and Discussion}

3.1. The Stress in AlGaN Barrier Layer Determined by NearResonant Raman Scattering. The temperature-dependent UV Raman spectra of $\mathrm{AlGaN} / \mathrm{GaN}$ heterostructure are shown in Figure 1. Compared to the visible Raman spectrum of AlGaN/GaN heterostructure [19], a new peak near $785 \mathrm{~cm}^{-1}$ occurs in the UV Raman spectrum. This peak corresponds to the $\mathrm{A}_{1}(\mathrm{LO})$ mode of the AlGaN layer according to the Alcomposition dependent $A_{1}(L O)$ phonon frequency [20]. As shown in Figure 1, the $A_{1}(\mathrm{LO})$ phonon mode of the AlGaN layer shows enhancement effect in intensity and red shift in frequency with increasing temperature.

The temperature dependence of the intensity of the $\mathrm{A}_{1}(\mathrm{LO})$ phonon mode in $\mathrm{AlGaN}$ can be explained by studying the resonant Raman scattering in the structure with varying temperatures. By solving the Schrodinger and Poisson equations self-consistently using the Silvaco Atlas software, we can get the band diagram of the structure with varying temperature. The band gaps of $\mathrm{AlGaN}$ and $\mathrm{GaN}$ in the temperature range of $80-600 \mathrm{~K}$ are shown in Figure 2. The band gap of the AlGaN barrier layer is closer to the excitation energy than that of the GaN layer in the whole temperature range. The resonant Raman scattering arises from the AlGaN barrier layer. The band gap of the AlGaN barrier layer decreases and becomes closer and closer to the excitation energy with the increasing temperature. So, the intensity of the $A_{1}(L O)$ phonon mode of $A l G a N$ increases with the increasing temperature. 


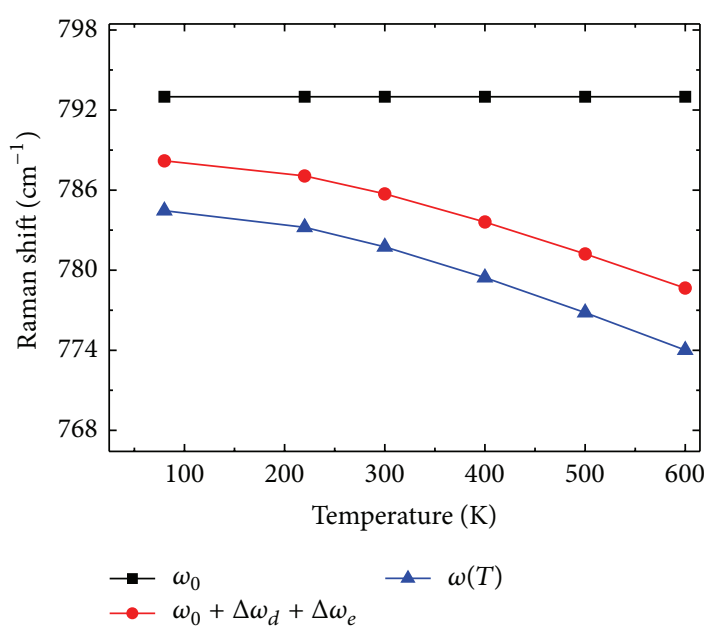

(a)

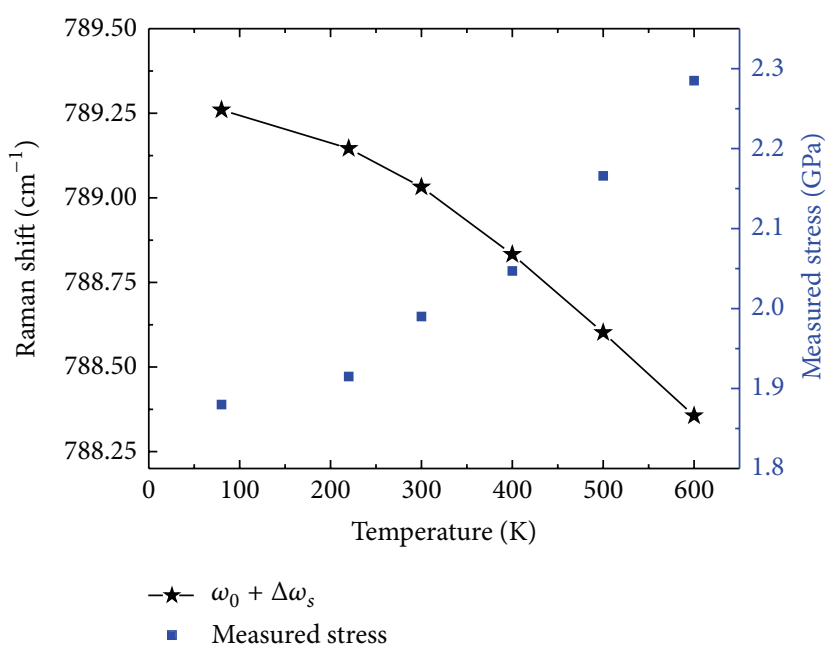

(b)

Figure 3: (a) Raman shift of $A_{1}$ (LO) mode of $A l G a N$ as a function of temperature together with the contribution of thermal expansion of lattice and phonon decay effects. (b) Temperature-dependent stress in AlGaN and its contribution to the Raman shift.

TABLE 1: Model parameters for AlGaN, GaN, and substrate [16].

\begin{tabular}{|c|c|c|c|c|c|c|c|c|}
\hline Material & $\theta_{1}(\mathrm{~K})$ & $\theta_{2}(\mathrm{~K})$ & $\theta_{3}(\mathrm{~K})$ & $\theta_{4}(\mathrm{~K})$ & $X_{1}\left(10^{-7} / \mathrm{K}\right)$ & $X_{2}\left(10^{-7} / \mathrm{K}\right)$ & $X_{3}\left(10^{-7} / \mathrm{K}\right)$ & $X_{4}\left(10^{-7} / \mathrm{K}\right)$ \\
\hline \multicolumn{9}{|l|}{$\overline{\mathrm{GaN}}$} \\
\hline$a$ & 75 & 581.25 & 1684.375 & & 0.487 & 52.152 & 4.21 & \\
\hline$c$ & 75 & 590.625 & 1675 & & 0.621 & 47.312 & 1.125 & \\
\hline \multicolumn{9}{|l|}{$\mathrm{AlN}$} \\
\hline$a$ & 125 & 600 & 1852.5 & & -4.348 & 44.074 & 35.056 & \\
\hline$c$ & 100 & 528.75 & 1723.75 & & -5.174 & 29.857 & 39.565 & \\
\hline \multicolumn{9}{|c|}{$\mathrm{Al}_{0.27} \mathrm{Ga}_{0.73} \mathrm{~N}$} \\
\hline$a$ & 88.5 & 586.313 & 1729.77 & & -0.0818 & 4.997 & 1.254 & \\
\hline$c$ & 81.75 & 573.919 & 1688.16 & & -0.0943 & 4.26 & 1.1504 & \\
\hline \multicolumn{9}{|l|}{ Sapphire } \\
\hline$a$ & 135 & 565.625 & 1231.25 & 5468.75 & 1.2176 & 53.401 & 35.613 & 23.661 \\
\hline$c$ & 135 & 598.438 & 1468.75 & 5198.438 & 2.856 & 72.079 & 23.202 & 29.087 \\
\hline
\end{tabular}

There are several reasons for the frequency shift of phonon mode with varying temperature. The anharmonicity of the crystal lattice gives rise to the thermal expansion of lattice and phonon decay $[21,22]$. The frequency shifts due to these two effects are denoted as $\Delta \omega_{e}(T)$ and $\Delta \omega_{d}(T)$, respectively. In an isotropic approximation, the term $\Delta \omega_{e}(T)$ is given by $[21,22]$

$$
\Delta \omega_{e}(T)=-\omega_{0} \gamma \int_{0}^{T}\left[\alpha_{c}(\widetilde{T})+2 \alpha_{a}(\widetilde{T})\right] d \widetilde{T},
$$

where $\alpha_{a}$ and $\alpha_{c}$ are the temperature-dependent thermal expansion coefficients along $a$ - and $c$-directions, $\omega_{0}$ is the harmonic frequency of the optical phonon mode, and $\gamma$ is the Grüneisen parameter. Here, the thermal expansion coefficient with variable temperature was described within multifrequency Einstein model [16]. Consider

$$
\alpha=\sum_{i=1}^{n} X_{i} \frac{\left(\theta_{i} / T\right)^{2} \exp \left(\theta_{i} / T\right)}{\left[\exp \left(\theta_{i} / T\right)-1\right]^{2}},
$$

where $X_{i}$ and $\theta_{i}$ are model parameters listed in Table 1 .
Taking into account symmetric decays of the zonecenter phonons into two phonons and three phonons with frequencies $\omega_{0} / 2$ and $\omega_{0} / 3$, respectively, the term $\Delta \omega_{d}(T)$ can be described by [23]

$$
\begin{aligned}
\Delta \omega_{d}(T)= & A\left[1+2 n\left(T, \frac{\omega_{0}}{2}\right)\right] \\
& +B\left[1+3 n\left(T, \frac{\omega_{0}}{3}\right)+3 n^{2}\left(T, \frac{\omega_{0}}{3}\right)\right],
\end{aligned}
$$

where $A$ and $B$ are constants and $n(T, \omega)=\left[\exp \left(\hbar \omega / k_{B} T\right)-\right.$ $1]^{-1}$ is the Bose-Einstein distribution function which describes the thermal occupation number of phonon states. The parameters $\gamma, \omega_{0}, A$, and $B$ for AlGaN are 1.56, $793 \mathrm{~cm}^{-1},-4.646 \mathrm{~cm}^{-1}$, and $-0.115 \mathrm{~cm}^{-1}$, respectively. The contributions of the thermal expansion of lattice and phonon decay effect to the frequency shift of $A_{1}(\mathrm{LO})$ mode in $\mathrm{AlGaN}$ are shown in Figure 3(a).

Besides the phonon frequency shift due to the thermal expansion of lattice and the decay of optical phonon into phonon with lower energy, the temperature-dependent stress 


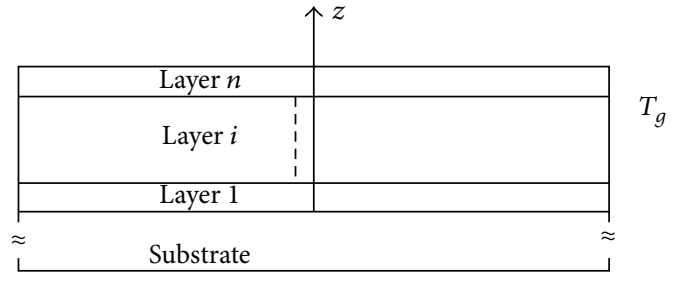

(a)

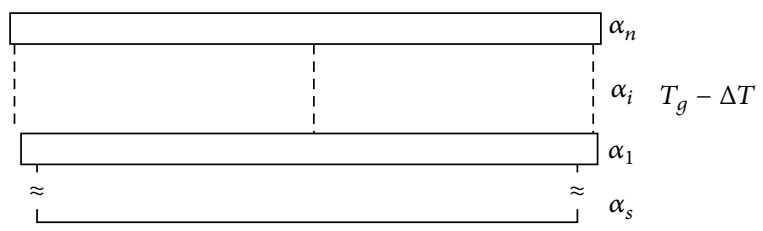

(b)

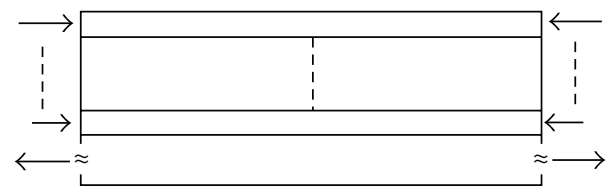

(c)

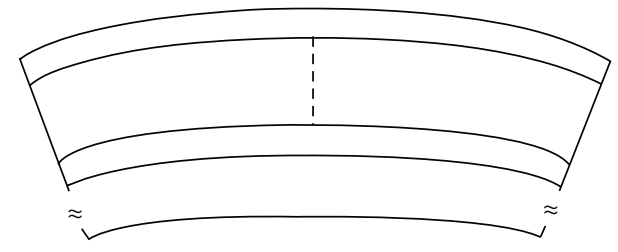

(d)

FIGURE 4: Schematic of the generation of thermal stress in multilayer structure.

in crystalline also contributes to the frequency shift, which is denoted as $\Delta \omega_{s}(T)$ [24]. Consider

$$
\Delta \omega_{s}(T)=2 \widetilde{a}_{\lambda} \sigma_{x x}+\widetilde{b}_{\lambda} \sigma_{z z} .
$$

For $\mathrm{A}_{1}(\mathrm{LO})$ mode in $\mathrm{Al}_{0.27} \mathrm{Ga}_{0.73} \mathrm{~N}$, the phonon deformation potentials $\widetilde{a}_{\lambda}, \widetilde{b}_{\lambda}$ equal 1.001 and $-1.576 \mathrm{~cm}^{-1} / \mathrm{GPa}$, respectively [24]. The temperature-dependent phonon frequency should be written as

$$
\omega(T)=\omega_{0}+\Delta \omega_{e}(T)+\Delta \omega_{d}(T)+\Delta \omega_{s}(T) .
$$

According to the measured $\omega(T)$ and the calculated $\Delta \omega_{e}(T)$ and $\Delta \omega_{d}(T)$ as shown in Figure 3(a), the temperaturedependent $\Delta \omega_{s}(T)$ and the corresponding stress in AlGaN can be obtained. The results are shown in Figure 3(b). The measured stress in $\mathrm{AlGaN}$ increased from $1.88 \mathrm{GPa}$ at $80 \mathrm{~K}$ to $2.28 \mathrm{GPa}$ at $600 \mathrm{~K}$.

3.2. Theoretical Calculation. In order to identify the accuracy of the stress determination in thin AlGaN barrier layer by analyzing near-resonant Raman spectroscopy, we calculate the temperature-dependent stress state of AlGaN layer theoretically by applying a stress model with multilayer structure. The total stress in the AlGaN barrier layer of AlGaN/GaN heterostructure grown on sapphire substrate consists of two parts: one is thermal stress due to thermal mismatch between $\mathrm{AlGaN}$ and the underlying GaN/substrate and the other is induced by LMM between $\mathrm{AlGaN}$ and GaN.

Figure 4 shows the analysis of thermal stress generated in multilayer structure [25]. An elastic multilayer structure at growth temperature $T_{g}$ is shown schematically in Figure 4(a), where $i$ denote layer number. When temperature decreases $\Delta T$, there are unconstrained strains in different layers. Hence, the free thermal strain, $\alpha_{i} \Delta T$, is generated in this layer $i$, as shown in Figure 4(b). Then, in order to achieve displacement compatibility, uniform tensile/compressive stresses are imposed on the individual layers (Figure 4(c)). Finally, the whole structure bends due to the asymmetric stresses in the multilayer structure (Figure 4(d)).

Based on the logical analysis described in Figure 4, the thermal stress in the AlGaN/GaN/sapphire structure can be calculated using the analytical model proposed by Hsueh and Evans [26] which decomposes thermal strain into a uniform component and a bending component. The thermal stress in $\mathrm{AlGaN}$ by taking a first-order approximation (i.e., ignoring terms with orders of $t_{i}$ higher than one) is expressed as follows [25]:

$$
\begin{array}{r}
\sigma_{\text {thermal }}=\int_{T_{g}}^{T} Y_{2}\left[\alpha_{a, s}-\alpha_{a, 2}+4 \frac{Y_{1} t_{1}\left(\alpha_{a, 1}-\alpha_{a, s}\right)}{Y_{s} t_{s}}\right. \\
\left.+4 \frac{Y_{2} t_{2}\left(\alpha_{a, 2}-\alpha_{a, s}\right)}{Y_{s} t_{s}}\right] d \widetilde{T},
\end{array}
$$

where the subscripts $s, 1$, and 2 denote the substrate, GaN, and AlGaN, respectively, $Y$ is biaxial modulus given in terms of elastic constants $C_{i j}$ as $Y=C_{11}+C_{12}-2 C_{13}^{2} / C_{33}$, and $t$ is layer thickness. Here, the elastic constants, biaxial modulus, and lattice constant of AlGaN are calculated from Vegard's law. The above parameters are listed in Table 2.

Based on the above analysis, the temperature-dependent thermal stress in $\mathrm{AlGaN}$ layer can be calculated numerically. The calculated result as shown in the insert of Figure 5 indicates that the biaxial compressive stress in $\mathrm{AlGaN}$ layer decreases with the increasing temperature in the temperature range of $80-600 \mathrm{~K}$ below growth temperature.

Besides the thermal stress, the stress due to LMM between $\mathrm{AlGaN}$ and $\mathrm{GaN}$ also contributes to the total stress in AlGaN. This stress can be calculated using the following equations [10]:

$$
\begin{gathered}
\varepsilon_{x x}=\frac{a_{1}-a_{2}}{a_{2}}=\frac{a_{1}}{a_{2}}-1, \\
\sigma_{\mathrm{LMM}}=\left(C_{11}+C_{12}-\frac{2 C_{13}^{2}}{C_{33}}\right) \varepsilon_{x x},
\end{gathered}
$$

where $a_{1}, a_{2}$ are lattice constants of strain-free $\mathrm{GaN}$ and $\mathrm{Al}_{0.27} \mathrm{Ga}_{0.73} \mathrm{~N}$ in $c$-plane, respectively. $C_{i j}$ is elastic constant of $\mathrm{Al}_{0.27} \mathrm{Ga}_{0.73} \mathrm{~N}$. These parameters are also listed in Table 2. The stress due to LMM between $\mathrm{AlGaN}$ and $\mathrm{GaN}$ is $3.272 \mathrm{GPa}$. The total stress in AlGaN which is the sum of $\sigma_{\text {thermal }}$ and $\sigma_{\text {LMM }}$ with varying temperature is also shown in Figure 5. 
TABLE 2: Parameters used in theoretical calculation.

\begin{tabular}{lcccccc}
\hline Material & $C_{11}(\mathrm{GPa})$ & $C_{12}(\mathrm{GPa})$ & $C_{13}(\mathrm{GPa})$ & $C_{33}(\mathrm{GPa})$ & $Y(\mathrm{GPa})$ & $t(\mu \mathrm{m})$ \\
\hline $\mathrm{GaN}^{\mathrm{a}}$ & 390 & 145 & 106 & 398 & 478.5 & 2 \\
$\mathrm{AlN}^{\mathrm{b}}$ & 410 & 149 & 99 & 389 & 508.6 & 3.206 \\
$\mathrm{Al}_{0.27} \mathrm{Ga}_{0.73} \mathrm{~N}$ & 395.5 & 146 & 104 & 395.6 & 486.7 & 0.025 \\
sapphire & 496 & 164 & 115 & 498 & 606.9 & 3.131 \\
\hline
\end{tabular}

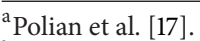

${ }^{\mathrm{b}}$ McNeil et al. [18].

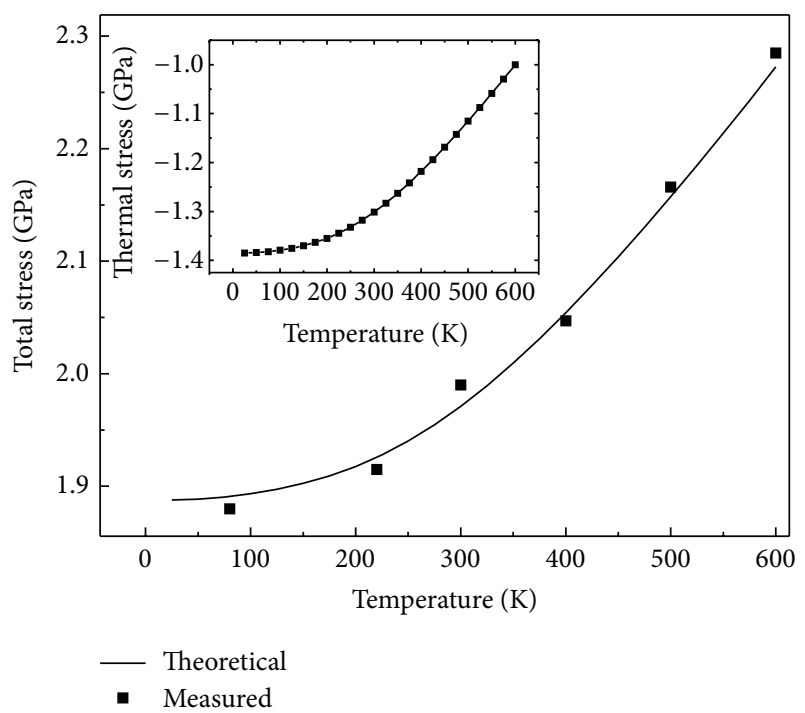

FIGURE 5: Temperature-dependent stress in AlGaN barrier layer determined by Raman scattering and theoretical calculation. The inset shows the temperature dependence of thermal stress in AlGaN.

The total stress increases from $1.89 \mathrm{GPa}$ at $80 \mathrm{~K}$ to $2.27 \mathrm{GPa}$ at $600 \mathrm{~K}$, which is consistent with the result obtained from near-resonant Raman scattering.

\section{Conclusions}

The temperature-dependent stress state in the AlGaN barrier layer of $\mathrm{AlGaN} / \mathrm{GaN}$ heterostructure was investigated by UV near-resonant Raman scattering. Strong scattering peak resulting from the $A_{1}(\mathrm{LO})$ phonon mode of $\mathrm{AlGaN}$ is observed under near-resonance condition. The temperaturedependent stress in the AlGaN layer determined by the resonance Raman spectra is consistent with the theoretical calculation result. This good agreement indicates that the UV near-resonant Raman scattering can be a direct and effective method to characterize the stress state in thin AlGaN barrier layer of AlGaN/GaN HEMT heterostructures.

\section{Conflict of Interests}

The authors declare that there is no conflict of interests regarding the publication of this paper.

\section{Acknowledgments}

This work was supported by the National 973 Project, China (2012CB619306, 2011CB301900), NSFC (nos. 61274075 and 61474060), NSF of Jiangsu Province, China (BK2011010), and Ph.D. Programs Foundation of Ministry of Education of China (20110091110032).

\section{References}

[1] U. K. Mishra, P. Parikh, and Y.-F. Wu, "AlGaN/GaN HEMTsan overview of device operation and applications," Proceedings of the IEEE, vol. 90, no. 6, pp. 1022-1031, 2002.

[2] M. A. Khan, Q. Chen, J. W. Yang, M. S. Shur, B. T. Dermott, and J. A. Higgins, "Microwave operation of GaN/AlGaN-doped channel heterostructure field effect transistors," IEEE Electron Device Letters, vol. 17, no. 7, pp. 325-327, 1996.

[3] Y.-F. Wu, A. Saxler, M. Moore et al., "30-W/mm GaN HEMTs by Field Plate Optimization," IEEE Electron Device Letters, vol. 25, no. 3, pp. 117-119, 2004.

[4] Y. Dora, A. Chakraborty, L. McCarthy, S. Keller, S. P. Denbaars, and U. K. Mishra, "High breakdown voltage achieved on AlGaN/GaN HEMTs with integrated slant field plates," IEEE Electron Device Letters, vol. 27, no. 9, pp. 713-715, 2006.

[5] X.-D. Wang, W.-D. Hu, X.-S. Chen, and W. Lu, "The study of self-heating and hot-electron effects for AlGaN/GaN doublechannel HEMTs," IEEE Transactions on Electron Devices, vol. 59, no. 5, pp. 1393-1401, 2012.

[6] N. Maeda, K. Tsubaki, T. Saitoh, and N. Kobayashi, "Hightemperature electron transport properties in $\mathrm{AlGaN} / \mathrm{GaN}$ heterostructures," Applied Physics Letters, vol. 79, no. 11, pp. 16341636, 2001.

[7] R. Gaska, Q. Chen, J. Yang, A. Osinsky, M. A. Khan, and M. S. Shur, "High-temperature performance of AlGaN/GaN HFET's on SiC substrates," IEEE Electron Device Letters, vol. 18, no. 10, pp. 492-494, 1997.

[8] T. Egawa, H. Ishikawa, M. Umeno, and T. Jimbo, "Recessed gate AlGaN/GaN modulation-doped field-effect transistors on sapphire," Applied Physics Letters, vol. 76, no. 1, pp. 121-123, 2000.

[9] O. Ambacher, J. Smart, J. R. Shealy et al., "Two-dimensional electron gases induced by spontaneous and piezoelectric polarization charges in N- And Ga-face AIGaN/GaN heterostructures," Journal of Applied Physics, vol. 85, no. 6, pp. 3222-3233, 1999.

[10] W. D. Hu, X. S. Chen, Z. J. Quan, C. S. Xia, W. Lu, and P. D. Ye, "Self-heating simulation of GaN-based metal-oxidesemiconductor high-electron-mobility transistors including hot electron and quantum effects," Journal of Applied Physics, vol. 100, no. 7, Article ID 074501, 2006. 
[11] N. Guo, W.-D. Hu, X.-S. Chen, L. Wang, and W. Lu, "Enhanced plasmonic resonant excitation in a grating gated field-effect transistor with supplemental gates," Optics Express, vol. 21, no. 2, pp. 1606-1614, 2013.

[12] M. K. Öztürk, H. Altuntaş, S. Çörekçi, Y. Hongbo, S. Özçelik, and E. Özbay, "Strain-stress analysis of AlGaN/GaN heterostructures with and without an AlN buffer and interlayer," Strain, vol. 47, no. 2, pp. 19-27, 2011.

[13] D. Chen, B. Shen, K. Zhang et al., "High-temperature characteristics of strain in AlGaN/GaN heterostructures," Japanese Journal of Applied Physics, vol. 45, no. 1, pp. 18-20, 2006.

[14] M. Kuball, S. Rajasingam, A. Sarua et al., "Measurement of temperature distribution in multifinger $\mathrm{AlGaN} / \mathrm{GaN}$ heterostructure field-effect transistors using micro-Raman spectroscopy," Applied Physics Letters, vol. 82, no. 1, pp. 124-126, 2003.

[15] R. J. T. Simms, J. W. Pomeroy, M. J. Uren, T. Martin, and M. Kuball, "Channel temperature determination in high-power AlGaN/GaN HFETs using electrical methods and Raman spectroscopy," IEEE Transactions on Electron Devices, vol. 55, no. 2, pp. 478-482, 2008.

[16] R. R. Reeber and K. Wang, "Lattice parameters and thermal expansion of important semiconductors and their substrates," MRS Proceedings, vol. 622, Article ID T6.35, 2000.

[17] A. Polian, M. Grimsditch, and I. Grzegory, "Elastic constants of gallium nitride," Journal of Applied Physics, vol. 79, no. 6, pp. 3343-3344, 1996.

[18] L. E. McNeil, M. Grimsditch, and R. H. French, "Vibrational spectroscopy of aluminum nitride," Journal of the American Ceramic Society, vol. 76, no. 5, pp. 1132-1136, 1993.

[19] D. J. Chen, B. Shen, X. L. Wu et al., "Temperature characterization of Raman scattering in an AlGaN/GaN heterostructure," Applied Physics A, vol. 80, no. 8, pp. 1729-1731, 2005.

[20] M. Kuball, "Raman spectroscopy of GaN, AlGaN and AlN for process and growth monitoring/control," Surface and Interface Analysis, vol. 31, no. 10, pp. 987-999, 2001.

[21] G. Irmer, M. Wenzel, and J. Monecke, "The temperature dependence of the $\mathrm{LO}(\Gamma)$ and $\mathrm{TO}(\Gamma)$ phonons in GaAs and InP," Physica Status Solidi (b), vol. 195, no. 1, pp. 85-95, 1996.

[22] J. Menéndez and M. Cardona, “Temperature dependence of the first-order Raman scattering by phonons in Si, Ge, and -Sn: anharmonic effects," Physical Review B, vol. 29, no. 4, pp. 20512059, 1984.

[23] M. Balkanski, R. F. Wallis, and E. Haro, "Anharmonic effects in light scattering due to optical phonons in silicon," Physical Review B, vol. 28, no. 4, pp. 1928-1934, 1983.

[24] J.-M. Wagner and F. Bechstedt, "Phonon deformation potentials of $\alpha-\mathrm{GaN}$ and -AlN: an ab initio calculation," Applied Physics Letters, vol. 77, no. 3, pp. 346-348, 2000.

[25] C. H. Hsueh, "Thermal stresses in elastic multilayer systems," Thin Solid Films, vol. 418, no. 2, pp. 182-188, 2002.

[26] C. H. Hsueh and A. G. Evans, "Residual stresses in metal/ ceramic bonded strips," Journal of the American Ceramic Society, vol. 68, no. 5, pp. 241-248, 1985. 

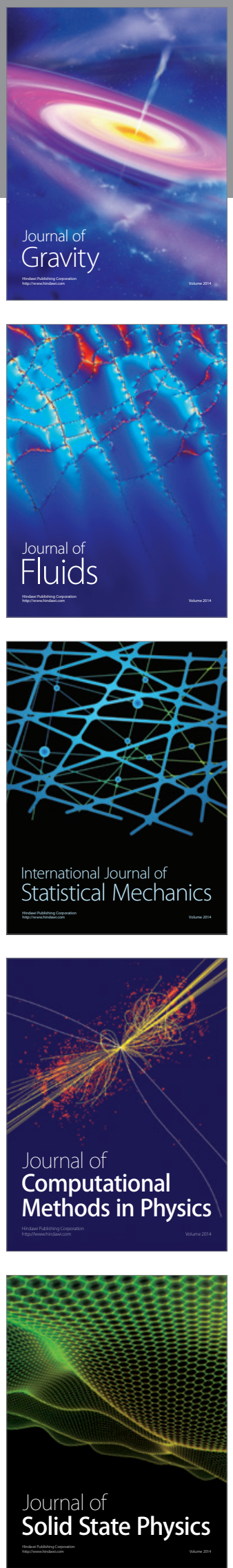

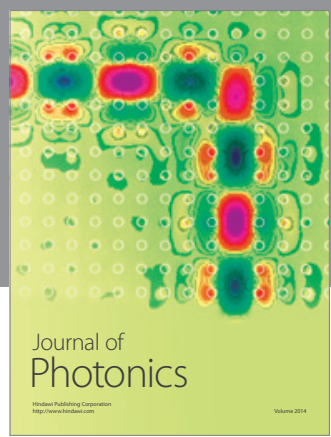

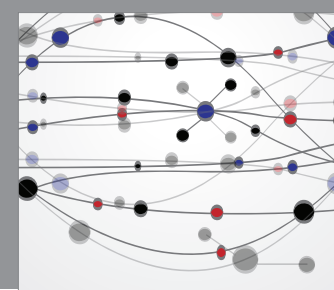

The Scientific World Journal

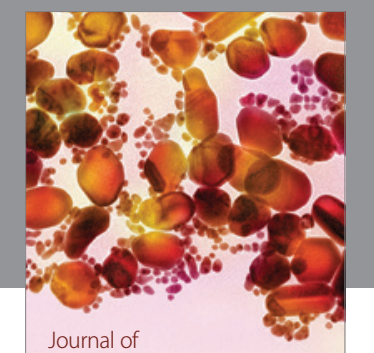

Soft Matter
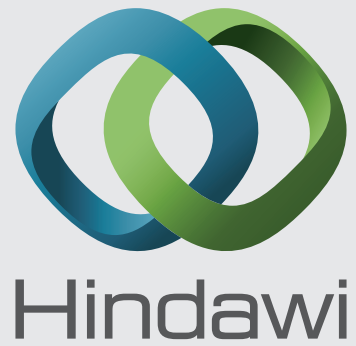

Submit your manuscripts at

http://www.hindawi.com
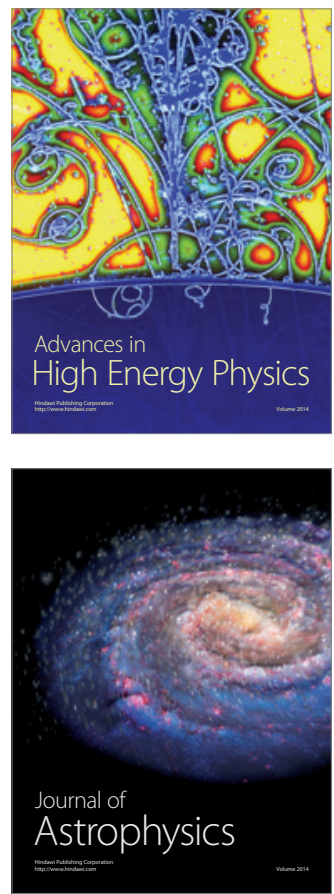
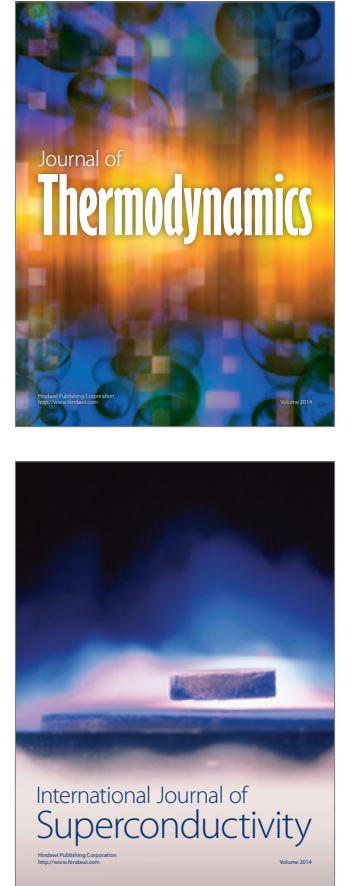
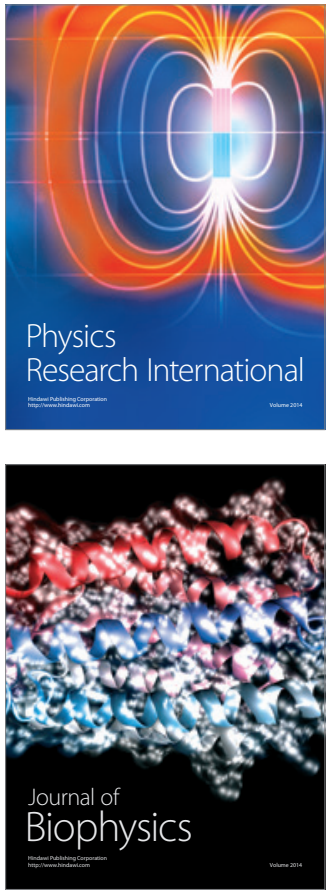
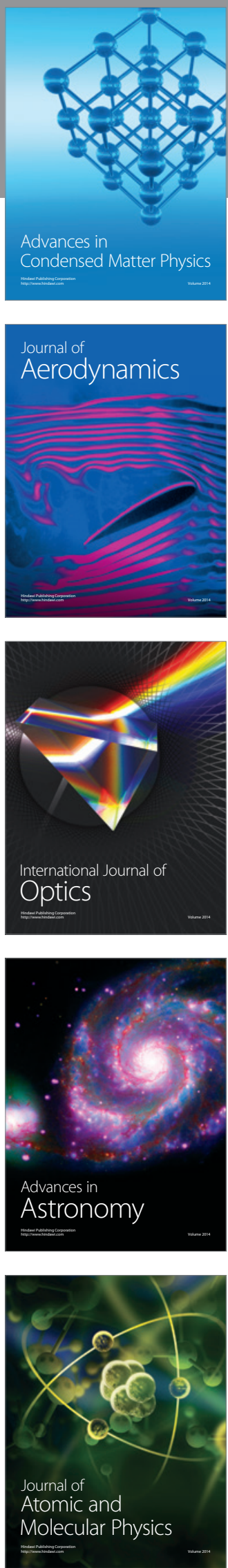